\title{
Pityriasis versicolor unter dem klinischen Bild einer Papillomatosis confluens et reticularis
}

\section{G. Wagner ${ }^{1}$ \\ C. Diaz $^{2}$ \\ W. Weyers ${ }^{2}$}

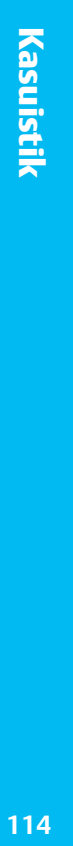

Tinea Versicolor Clinically Presenting as Confluent and Reticulated Papillomatosis

\section{Zusammenfassung}

Bei einer 16-jährigen Patientin fanden sich nebeneinander die unterschiedlichen klinisch-morphologischen Befunde der Pityriasis versicolor und der Papillomatosis confluens et reticularis. Die zunächst eingeleitete orale Therapie mit Minocyclin führte zu keiner Rückbildung der Befunde. Erst nach anschließender topischer Behandlung mit Ciclopirox kam es innerhalb weniger Tage zu einer vollständigen Abheilung. Der vor Beginn der Behandlung in den klinisch-morphologisch unterschiedlichen Läsionen erfolgte Nachweis von Pityrosporum ovale gelang nach Abschluss der Therapie nicht mehr. Die Beurteilung der klinischen, mykologischen und histopathologischen Befunde sowie das identische Verhalten der zunächst getrennt betrachteten Dermatosen auf die unterschiedlichen Therapieverfahren lassen den Schluss zu, dass in dem hier vorgestellten Fall die Papillomatosis confluens et reticularis als klinische Manifestationsform der Pityriasis versicolor verstanden werden kann.

\section{Abstract}

A 16 year old female patient presented herself with the two clinical distinct morphological patterns of tinea versicolor and confluent and reticulated papillomatosis. Initial treatment with oral minocycline was ineffective. Subsequent topical treatment with ciclopirox yielded complete healing within a few days. Pityrosporum ovale could be demonstrated in both types of lesions before therapy, but not after treatment. Initially, the dermatoses were interpreted as separate types of skin disease. Assessment of clinical, mycological, and histopathological findings as well as identical responses to the different modalities of treatment led to the conclusion that, in this case, the clinical picture of confluent and reticulated papillomatosis represented a manifestation of tinea versicolor.

\section{Einleitung}

Die 1927 von Gougerot und Carteaud erstmals beschriebene Papillomatosis confluens et reticularis ist eine selten beobachtete Dermatose, die durch einen anfänglich progredienten, später chronisch-stationären Verlauf gekennzeichnet ist [1]. Bevorzugt betroffen sind Jugendliche und junge Erwachsene, wobei die Frage umstritten ist, ob Frauen häufiger erkranken als Männer. Dabei konnte eine von Groh und Schnyder angegebene Geschlechtspräferenz zugunsten der Frauen durch Lee et al. nicht bestätigt werden [2,3].Zu den Prädilektionsstellen der Papillomatosis confluens et reticularis zählen die Intermammär- und Interskapularregion mit einer hier typischerweise rhomboidalen, kraniokaudalen Ausbreitung der Morphen sowie die lateralen Anteile des Thorax, die Achseln und der Hals. Die klinische Morphologie der Dermatose ist gekennzeichnet durch braun bis dunkelbraun pigmentierte, maximal linsengroße Papeln, die zentral zu größeren Arealen konfluieren, während sie in der Peripherie der Herde ein netzförmiges Muster ausbilden [2]. Subjektive Missempfindungen bestehen im Allgemeinen nicht. Obwohl der Verlauf und das klini-

Institutsangaben

${ }^{1}$ Hautklinik Zentralkrankenhaus Bremerhaven, (Chefarzt: Dr. G. Wagner)

2 Einsendungslabor für Dermatopathologie, Freiburg

Korrespondenzadresse

Dr. G. Wagner · Hautklinik Zentralkrankenhaus · Reinkenheide · Postbrookstr. $103 \cdot 27574$ Bremerhaven

Bibliografie

Akt Dermatol 2004; 30: 114-119 ㄷ Georg Thieme Verlag KG Stuttgart · New York · DOI 10.1055/s-2004-814419

ISSN 0340-2541 
sche Bild der Papillomatosis confluens et reticularis als charakteristisch bezeichnet werden können, wurde die Eigenständigkeit der Dermatose immer wieder infrage gestellt [4-7]. Die phänomenologischen Ähnlichkeiten zwischen der Papillomatosis confluens et reticularis einerseits sowie der Pityriasis versicolor bzw. der Acanthosis nigricans andererseits und das vereinzelt beschriebene gemeinsame Auftreten der genannten Dermatosen haben zur Diskussion über die Nosologie der Papillomatosis confluens et reticularis genauso beigetragen wie der Umstand, dass Ätiologie und Pathogenese der Papillomatosis confluens et reticularis bis heute nicht geklärt werden konnten.

Die hier vorgestellte Kasuistik unterstreicht das Problem der nosologischen Einordnung der Papillomatosis confluens et reticularis und wirft gleichzeitig die Frage auf, ob möglicherweise ätiologisch unterschiedliche Erkrankungen das klinische Bild der Papillomatosis confluens et reticularis hervorrufen können.

\section{Kasuistik}

\section{Anamnese}

Bei der 16-jährigen Patientin waren erstmals vor 2 Jahren nicht juckende Hautveränderungen am vorderen Dekolleté und am Hals aufgetreten, die anschließend langsam an Ausdehnung zunahmen. Die Behandlung war mit verschiedenen glukokortikoid-haltigen Salben sowie zwischenzeitlich auch mit Harnstoff-, Benzoylperoxid- und Teerrezepturen erfolgt, ohne dass eine Rückbildung der Befunde erreicht werden konnte.

Die allgemeine Anamnese der Patientin war unauffällig. Dabei ergaben sich insbesondere keine Hinweise für bekannt gewordene internistische Stoffwechselerkrankungen.

\section{Dermatologischer Befund}

Am vorderen Dekolleté sowie am Hals ventral und lateral bds. flächenhaft angeordnete, zentral zu größeren, homogen wirkenden Arealen konfluierende, peripher leistenartig angeordnete, hier netzgitterartige Strukturen bildende, braun pigmentierte Papeln, teilweise mit verruköser, teilweise mit lichenoider Oberfläche. Ferner inter- und submammär münzgroße, vereinzelt konfluierende, scharf begrenzte, bräunlich-rötlich pigmentierte Maculae. Positives Hobelspanphänomen (Abb.1-4). Mit Ausnahme einer Adipositas unauffälliger Befund bei der allgemeinkörperlichen Untersuchung.

\section{Befunde diagnostischer Untersuchungen Histopathologischer Befund}

Starke Auffaltung der Epidermis, die von einer breiten, korbgeflechtartig strukturierten Orthohyperkeratose bedeckt wird (Abb. 5). In der Hornschicht sind bereits in der HE-Färbung zahlreiche Sporen nachweisbar, die in der PAS-Färbung noch deutlicher dargestellt werden (Abb. 6). Abgesehen vom Pilznachweis, der papillomatösen Auffaltung und der lockeren Orthohyperkeratose weist die Epidermis keine pathologischen Veränderungen auf. Die Kongorot-Färbung zeigt keine Amyloid-Ablagerungen in den Papillenspitzen. In der oberen Dermis findet sich ein nur minimales perivaskuläres Lymphozyteninfiltrat.

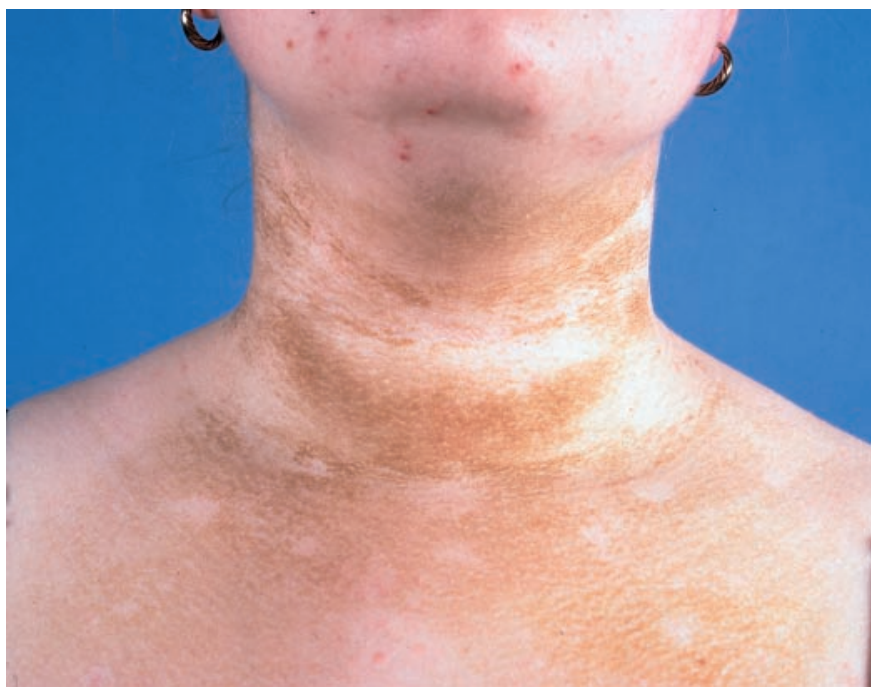

Abb. 1 Papillomatosis confluens et reticularis ventraler Hals und vorderes Dekolleté.

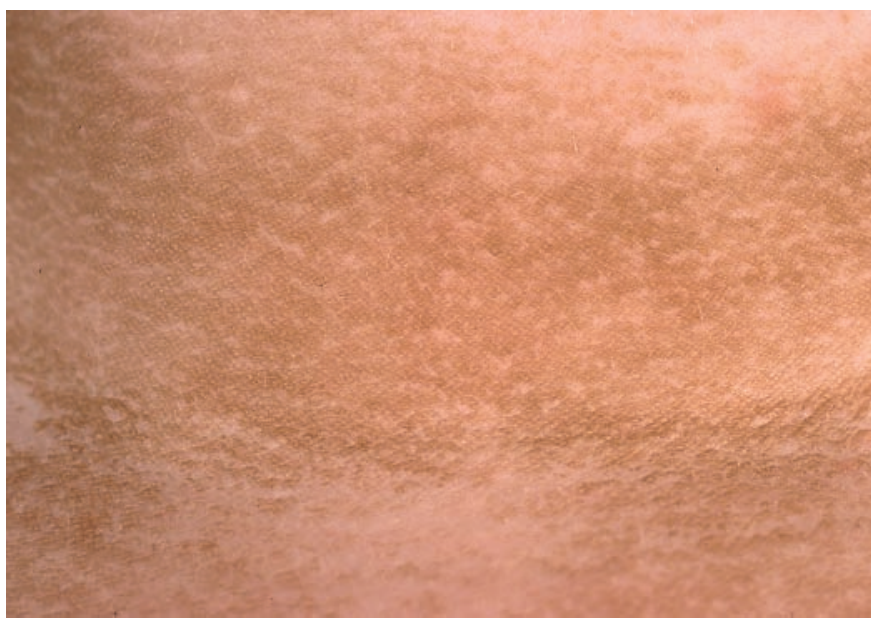

Abb. 2 Detailaufnahme ventraler Hals: Konfluierende, pigmentierte Papeln.

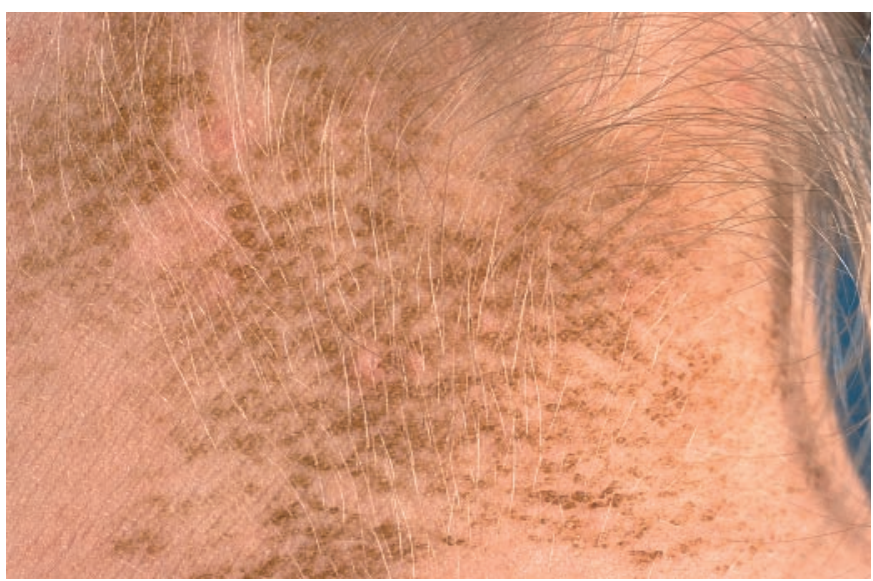

Abb. 3 Detailaufnahme lateraler Hals: Retikulär angeordnete, pigmentierte Papeln. 


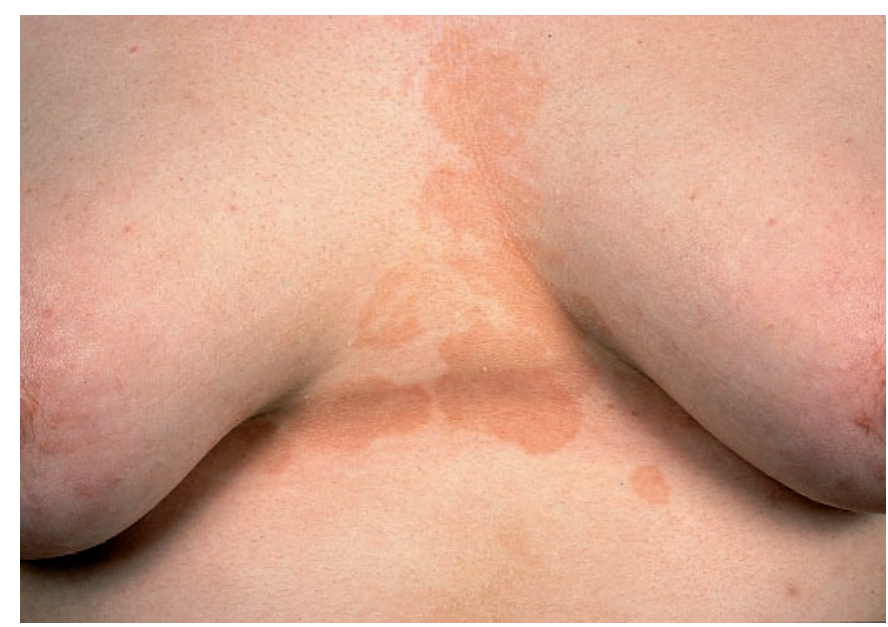

Abb. 4 Pityriasis versicolor: Makulöse Läsionen inter- und submammär.

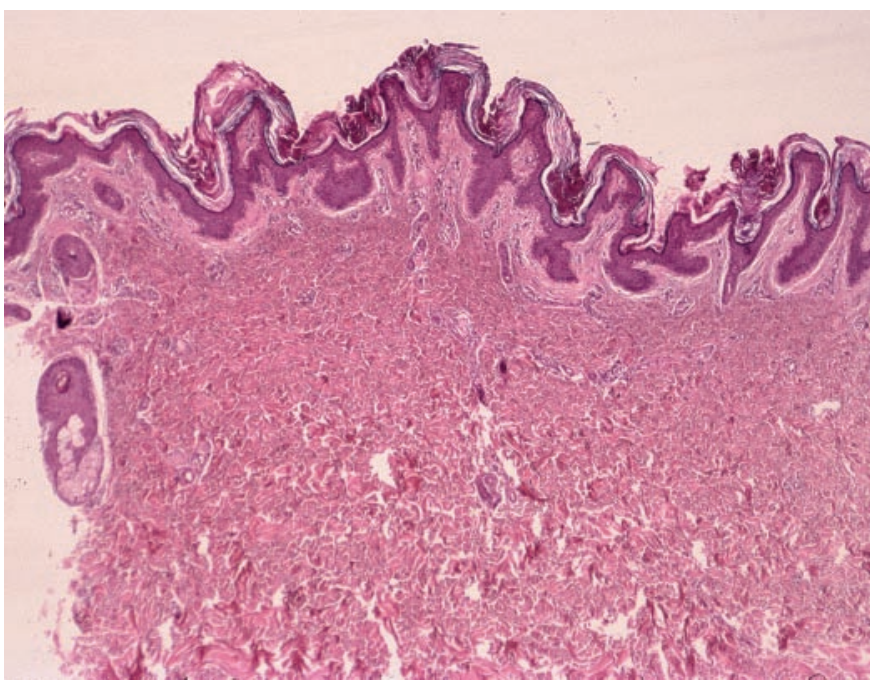

Abb. 5 Typischer histopathologischer Befund der Papillomatosis confluens et reticularis: Die Epidermis ist papillomatös aufgefaltet und wird von einer lockeren Orthohyperkeratose bedeckt. Die Reteleisten sind zum Teil verlängert, ansonsten ist die Epidermis jedoch nicht verbreitert. Kein nennenswertes Entzündungsinfiltrat (HE, 1:40). Kongorotfärbung negativ.

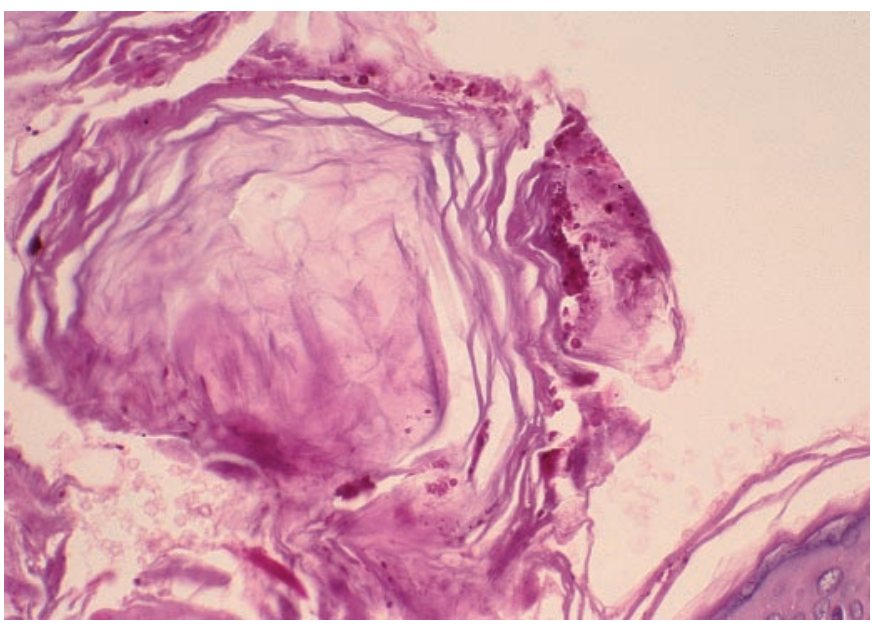

Abb. 6 Innerhalb der verbreiterten, korbgeflechtartig aufgebauten Hornschicht finden sich zahlreiche Sporen (PAS, 1:400).

\section{Mikrobiologische Befunde}

\section{Mykologie}

Nativpräparate vom lateralen Hals und intermammär: Nachweis von in Haufen liegenden, dickwandigen Sporen und einzelnen kurzen Myzelfäden.

Kulturen von gleichen Lokalisationen (Sabouraud-Glukose-Agar mit Oliven-Öl): Wachstum von Pityrosporum ovale.

\section{Bakteriologie}

Kultur vom lateralen Hals: Staph. aureus. Stamm unter anderem gegen Minocyclin empfindlich.

\section{Laborbefunde}

Blut- und Differenzialblutbild, BSG, CRP, Lebertransaminasen, nierenpflichtige Substanzen, Blutfette und Eiweißelektrophorese ohne pathologische Befunde. Unauffälliges Blutzuckertagesprofil, euthyreote Stoffwechsellage.

\section{Therapie und Verlauf}

Die Behandlung erfolgte zunächst mit $2 \times 50 \mathrm{mg}$ Minocyclin täglich über einen Zeitraum von 4 Wochen, ohne dass eine Rückbildung der Befunde festgestellt werden konnte. Daraufhin wurde die Therapie mit Minocyclin abgebrochen. Die anschließend durchgeführte Behandlung mit Ciclopirox (Batrafen Creme ${ }^{\circledR}$ ) führte bei täglich zweimaliger Anwendung innerhalb von $10 \mathrm{Ta}-$ gen zu einer vollständigen Rückbildung des gesamten klinischen Befundes. Nach Abschluss der lokalen Behandlung wurden erneut mykologische Kulturen vom Hals und der intermammären Region angelegt. In beiden Lokalisationen konnte Pityrosporum ovale nicht mehr nachgewiesen werden.

\section{Diskussion}

Die bei der hier vorgestellten Patientin am vorderen Dekolleté und am Hals aufgetretenen Hautveränderungen wurden aufgrund der klinischen Morphologie und der gleichzeitig erhobenen histopathologischen Befunde als Papillomatosis confluens et reticularis eingeordnet. Dabei war das klinische Bild durch deutlich pigmentierte, zentral konfluierende, in der Peripherie hingegen netzgitterartig angeordnete Papeln gekennzeichnet, während bei der histopathologischen Untersuchung die für die Diagnose ebenfalls typischen Befunde einer Papillomatose und lockeren Orthohyperkeratose erhoben werden konnten. Die darüber hinaus inter- und submammär vorhandenen hellbraun pigmentierten Maculae wurden als typischer klinischer Befund einer Pityriasis versicolor gewertet und zunächst als zusätzlich bestehende zweite Dermatose angesehen. Da die Papillomatosis confluens et reticularis augenscheinlich im Vordergrund der klinischen Symptomatik stand, wurde eine orale Behandlung mit Minocyclin eingeleitet, auf deren Wirksamkeit in verschiedenen Kasuistiken hingewiesen worden ist [8-11]. Überraschenderweise führte die orale Minocyclin-Therapie zu keiner Besserung des klinischen Befundes. Im Hinblick auf den zwischenzeitlich auch kulturell geführten Nachweis von Pityrosporum ovale wurde die Behandlung auf die lokale Anwendung von Ciclopirox umgestellt, woraufhin der gesamte dermatologische Befund innerhalb weniger Tage vollständig abheilte und darüber hinaus Pityrosporum ovale nicht mehr nachgewiesen werden konnte. Auf- 
grund dieser Befunde haben wir im vorliegenden Fall die Papillomatosis confluens et reticularis als eine klinische Manifestation der Pityriasis versicolor eingeordnet. Die Papillomatosis confluens et reticularis konnte bei unserer Patientin schlüssig auf eine Infektion mit Pityrosporum ovale zurückgeführt werden.

Die Pityriasis versicolor, die klassische Manifestation einer durch Pityrosporum ovale ausgelösten Epidermomykose, zeigt sich üblicherweise in Form scharf begrenzter makulöser Läsionen, die sich im Vergleich zur umgebenden Haut durch hellere, dunkler pigmentierte oder erythematöse Farbtöne auszeichnen. Zu den Prädilektionsstellen der Pityriasis versicolor zählen die vordere und hintere Schweißrinne, eine Topografie, die auch in ihrer flächenhaften rhomboidalen Ausbreitung mit den typischen Befunden der Papillomatosis confluens et reticularis übereinstimmt. Auch das bevorzugte Alter der Patienten ist vergleichbar. Sowohl die Pityriasis versicolor als auch die Papillomatosis confluens et reticularis werden überwiegend bei Jugendlichen und jungen Erwachsenen im Alter zwischen 15 und 25 Jahren erstmals beobachtet $[2,12]$. Neben der makulösen Form, die am häufigsten beobachtet wird, wurden bei der Pityriasis versicolor auch andere, weniger bekannte klinische Variationen beschrieben wie z. B. die pseudoatrophische oder pseudoanetodermische Form der Pityriasis versicolor $[13,14]$. Darüber hinaus sind in der Literatur vereinzelt papulöse Morphen der Pityriasis versicolor beschrieben worden. Das daraus resultierende klinische Bild der Erkrankung wurde von den Autoren übereinstimmend als Papillomatosis confluens et reticularis bezeichnet [5,12,15-19]. So beobachteten Kamalam und Thambiah in einer Gruppe von 200 indischen Patienten mit einer Pityriasis versicolor bei $5 \%$ der Betroffenen eine Papillomatosis confluens et reticularis. Bei einer anderen Untersuchung, die ebenfalls in Indien durchgeführt wurde, fanden dieselben Autoren bei 2446 an einer Pityriasis versicolor erkrankten Patienten in 1,6\% der Fälle die typischen klinischen Befunde der Papillomatosis confluens et reticularis [12,17]. Dass die Papillomatosis confluens et reticularis als klinische Manifestationsform der Pityriasis versicolor somit ganz offensichtlich besonders häufig in tropischen bzw. subtropischen Regionen beobachtet wurde, ist vermutlich jedoch nur auf den Umstand zurückzuführen, dass die Prävalenz der Pityriasis versicolor in warmen Regionen mit 30-50\% insgesamt deutlich höher ist als in Nordeuropa, wo mit einer entsprechenden Häufigkeit von weniger als einem Prozent gerechnet werden muss [20]. Im Übrigen konnten einzelne Autoren auch über eine Rückbildung der Papillomatosis confluens et reticularis berichten, nachdem eine Behandlung mit Selendisulfid durchgeführt worden war $[5,18]$. Histopathologische Untersuchungen der Pityriasis versicolor in ihrer häufigsten makulösen Form zeigen eine geringgradige Orthohyperkeratose und manchmal ein diskretes, überwiegend lymphozytäres Infiltrat im oberen Korium [20]. Interessanterweise konnten Janaki et al. bei einem Patienten mit papulösen Morphen einer Pityriasis versicolor eine histopathologisch ausgeprägte Papillomatose beschreiben, wie sie nach Einschätzung der Autoren für eine Papillomatosis confluens et reticularis typisch ist [16].

In Ergänzung zu unserem eigenen Fall belegen somit auch die in der Literatur beschriebenen klinischen und histopathologischen Befunde die Auffassung, dass die Papillomatosis confluens et reticularis in Einzelfällen als klinische Manifestationsform der Pi- tyriasis versicolor und damit als Folge einer Infektion mit Pityrosporum ovale gewertet werden kann. Andererseits besteht jedoch kein Zweifel, dass diese Auffassung keineswegs eine grundsätzliche Gültigkeit besitzt. In verschiedenen kasuistischen Beiträgen zur Papillomatosis confluens et reticularis konnte der Nachweis von Pityrosporum ovale nicht geführt werden. Bei diesen Patienten blieben auch antimykotische Therapieverfahren ohne Wirkung, während anschließend verordnete orale Behandlungen mit verschiedenen Antibiotika, wie z.B. Minocyclin, Azithromycin und Cephalosporinen, zur Abheilung der jeweils vorliegenden Papillomatosis confluens et reticularis führten [9,10,21]. Andere Autoren haben bei den von ihnen beobachteten Patienten eine ätiologische Bedeutung von Pityrosporum ovale ebenfalls verneint, obwohl der Erreger nachweisbar war. So fanden Bonczkowitz et al. zwar eine Besiedlung der Papillomatosis confluens et reticularis ihrer Patientin, die durchgeführten lokalen Behandlungen mit Ketoconazol und Ciclopirox führten jedoch zu keiner Rückbildung der Dermatose. Auch in diesem Fall erbrachte erst eine orale Therapie mit Minocyclin den gewünschten Erfolg [8]. Im Zusammenhang mit dem fehlenden Nachweis von Pityrosporum ovale ist die Vermutung geäußert worden, dass der Erreger zwar ursächlich für die Auslösung der Papillomatosis confluens et reticularis verantwortlich sein könnte, z. B. als Auslöser einer abnormen epidermalen Reaktion, andererseits für die Persistenz der Hautveränderungen jedoch nicht unbedingt weiter vorhanden sein müsse [22]. Diese Hypothese erscheint aber wenig überzeugend, da sie einerseits nicht erklärt, warum eine üblicherweise chronisch-persistierende Besiedlung der Haut mit Pityrosporum ovale plötzlich abheilen sollte, und andererseits auch keine Antwort auf die Frage anbietet, auf welche Weise die genannten, pharmakologisch unterschiedlich wirksamen Antibiotika eine Rückbildung der histopathologisch nachweisbaren Papillomatose bewirken könnten. Als möglicher Wirkmechanismus für Tetrazykline wurde ein antiproliferativer Effekt durch Inhibition der Proteinkinase $C$ beschrieben, der jedoch bei Azithromycin und Cephalosporinen nicht bekannt ist [23]. Dass das klinische und histopathologische Bild der Papillomatosis confluens et reticularis sowohl durch eine oberflächliche Pilzinfektion als auch durch eine bakterielle Infektion induziert werden kann und den jeweiligen Therapieerfolgen ein direkter antimikrobieller Wirkungsmechanismus zugrunde liegt, ist demgegenüber wahrscheinlicher.

Neben der Diskussion über die Bedeutung von Pityrosporum ovale für die Entstehung der Papillomatosis confluens et reticularis sind weitere ätiopathogenetische Überlegungen diskutiert worden. Hierzu zählen endokrinologische Erkrankungen - vermutet wurden Beziehungen zum Diabetes mellitus, zu Störungen der Schilddrüsenfunktion und zur Adipositas - sowie möglicherweise genetisch bedingte Veränderungen der Keratinisierung $[24,25]$. Vereinzelt elektronenmikroskopisch nachgewiesene Befunde, z.B. morphologische Veränderungen der Korneozyten, konnten bei späteren Untersuchungen nicht nachvollzogen werden $[10,26]$. Wegen der klinischen und histopathologischen Ähnlichkeit der Papillomatosis confluens et reticularis mit der Akanthosis nigricans wurden Beziehungen zwischen diesen beiden Erkrankungen postuliert. Die Pseudoacanthosis nigricans wird typischerweise bei jungen, stets übergewichtigen Patienten beobachtet [27,28]. Dabei könnte die mit der Adipositas verbundene Hyperinsulinämie durch vermehrte Aktivierung des Insu- 
lin-like Growth-Faktors zu einer Stimulierung von Keratinozyten und dermalen Fibroblasten führen, die das klinische Bild der Pseudoacanthosis nigricans auslösen könnten [7,29]. Dieses Bild ist gekennzeichnet durch leistenartig angeordnete Papeln, die sich bevorzugt im Bereich der intertriginösen Räume, am Hals und am Nacken finden $[27,28]$. Treten die Morphen auch ventral oder dorsal am Stamm auf, entspricht das daraus resultierende klinische Bild der Pseudoacanthosis nigricans dem typischen Befund der Papillomatosis confluens et reticularis [5,7]. Da auch die histopathologischen Befunde beider Dermatosen identisch sind, was im Übrigen für alle Formen der Acanthosis nigricans gilt, wurde die Entität der Papillomatosis confluens et reticularis von verschiedenen Autoren bezweifelt [4, 7,28,30]. Im Gegensatz hierzu verweisen andere Autoren auf die ihrer Meinung nach doch unterschiedlichen Lokalisationen beider Dermatosen, das fehlende retikuläre Muster der Papeln bei der Pseudoacanthosis nigricans und auf die unterschiedliche therapeutische Wirkung einer Gewichtsreduktion [2,6,10,31]. Während eine deutliche Abnahme des Körpergewichtes bei der stets an eine Adipositas gebundene Pseudoacanthosis nigricans zur Rückbildung dieser Dermatose führen soll, sei dies bei der häufig auch ohne Adipositas vorkommenden Papillomatosis confluens et reticularis nicht der Fall $[32,33]$. Ob die aufgeführten klinischen Unterschiede jedoch ausreichen, um von nosologisch getrennten Erkrankungen ausgehen zu können, bleibt zweifelhaft. Unter der Vorstellung, dass der Papillomatosis confluens et reticularis eine Keratinisierungsstörung zugrunde liegt, sind wiederholt systemische Behandlungsversuche mit Isotretinoin und Etretinat erfolgreich durchgeführt worden. Nach Abbruch der Behandlungen kam es in der Mehrzahl der Fälle zu Rezidiven der Dermatose [24,34-36]. Der therapeutische Einfluss der Retinoide spricht zwar für eine pathogenetisch bedeutsame Keratinisierungsstörung, lässt andererseits jedoch keine Rückschlüsse auf die Ätiologie der Papillomatosis confluens et reticularis zu. Das unterschiedliche Ansprechen auf die verschiedenen Therapieverfahren sowie auch die teilweise widersprüchlichen Ergebnisse beim Einsatz gleicher Wirkstoffgruppen, z. B. bei den Antimykotika, lassen es offensichtlich erscheinen, dass der Papillomatosis confluens et reticularis verschiedene ätiologische Faktoren zugrunde liegen. Die Papillomatosis confluens et reticularis wäre demnach vielen anderen Hautkrankheiten an die Seite zu stellen, bei denen unterschiedliche Auslöser zu einem klinisch und histopathologisch charakteristischen Krankheitsbild führen.

Unabhängig von den dargestellten Problemen der Ätiologie, der Pathogenese und der nosologischen Stellung empfiehlt sich für die Behandlung der Papillomatosis confluens et reticularis ein den erhobenen Befunden angepasstes Vorgehen. Beim Nachweis von Pityrosporum ovale sollte zunächst eine lokale, bei sehr ausgedehnten Befunden eventuell auch orale antimykotische Therapie erfolgen. Führt diese Behandlung zu keiner Abheilung oder ist Pityrosporum ovale weder im Nativpräparat noch in der Kultur nachweisbar, ist eine orale Behandlung mit Minocyclin zu empfehlen. Sollte auch diese Therapie erfolglos bleiben, ist ein Behandlungsversuch mit Retinoiden gerechtfertigt.

\section{Literatur}

${ }^{1}$ Gougerot H, Carteaud A. Papillomatose pigmentée innominée. Bull Soc Fr Dermatol Syph 1927; 34: 719-721

${ }^{2}$ Groh V, Schnyder UW. Nosologie der Papillomatose papuleuse confluente et réticulée (Gougerot-Carteaud). Hautarzt 1983; 34: 81 - 86

${ }^{3}$ Lee MP, Stiller MJ, McClain SA, Shupack JL, Cohen DE. Confluent and reticulated papillomatosis: Response to high dose isoretinoin therapy and reassessment of epidemiologic data. J Am Acad Dermatol 1994; 31: $327-331$

${ }^{4}$ Kesten B, James HD. Pseudoatrophoderma colli, acanthosis nigricans, and confluent and reticulated papillomatosis. Arch Dermaol 1957; 75: $525-542$

${ }^{5}$ Roberts S, Lachapelle JM. Confluent and reticulate papillomatosis (Gougerot-Carteaud) and Pityrosporum orbiculare. Br J Dermatol 1969; 81: $841-845$

${ }^{6}$ Sau P, Lupton GP. Reticulated truncal pigmentation. Arch Dermatol 1988; 124: 1272 - 1275

${ }^{7}$ Wilhelm KP, Tronnier M, Wolff HH. Pseudoacanthosis nigricans unter dem klinischen Erscheinungsbild einer Papillomatosis confluens et reticularis (Gougerot und Carteaud). Hautarzt 1993; 44: 598 - 601

${ }^{8}$ Bonczkowitz M, Weyers W, Schill WB. Papillomatosis confluens et reticularis Gougerot-Carteaud - erfolgreiche Behandlung mit Minocyclin. H u G 1996; 71: 838-840

${ }^{9}$ Jürgens M, Schell H, Simon M, Schuler G. Papillomatosis confluens et reticularis. Akt Dermatol 1999; 25: 237-239

${ }^{10}$ Kretzschmar L, Metze D, Vehring KH, Bonsmann G. Papillomatosis confluens et reticularis. Hautarzt 1996; 47: 209-213

${ }^{11}$ Sassolas B, Plantin P, Guillet G. Confluent and reticulated papillomatosis: Treatment with minocycline. J Am Acad Dermatol 1992; 26 ; $501-502$

12 Chetty GN, Kamalam A, Thambiah AS. Pityriasis versicolor - a study of 200 cases in a tropical skin clinic. Mykosen 1979; 22: 234-246

${ }^{13}$ Runne U. Pseudo-Anetodermie im Bereich einer mit Corticosteroidexterna behandelten Pityriasis versicolor (2 Fälle). Z Hautkr 1975; 50: 449-451

${ }^{14}$ Wagner G, Lubach D. Pityriasis versicolor pseudoatrophicans. Eine Fallbeschreibung. Z Hautkr 1986; 62: 321 - 324

${ }^{15}$ Allen HB, Charles CR, Johnson BL. Hyperpigmented tinea versicolor. Arch Derm 1976; 112: 1110 - 1113

${ }^{16}$ Janaki C, Sentamilselvi G, Janaki VR, Boopalraj JM. Unusual observations in the histology of pityriasis versicolor. Mycopath 1997; 139: $71-74$

${ }^{17}$ Kamalam A, Thambiah AS. A study of 3891 cases of mycoses in the tropics. Sabouraudia 1976; 14: 129-148

18 Nordby CA, Mitchell AJ. Confluent et reticulated papillomatosis responsive to selenium sulfide. Int J Dermatol 1986; 25: 194-199

${ }^{19}$ Vanbreuseghem R, Randjamdiche M. Introduction to the epidemiology of pityriasis versicolor. Mykosen 1978; 1: 133-136

20 Balus L, Grigoriu D. Pityriasis versicolor. hbo-druck, Einhausen: 1982; 1. Aufl.

${ }^{21}$ Weigl LB, Beham A, Schnopp C, Möhrenschlager M, Abeck D. Papillomatosis confluens et reticularis. Erfolgreiche Therapie mit Azithromycin. Hautarzt 2001; 52: $947-949$

22 Yesudian P, Kamalam S, Razack A. Confluent et reticulated papillomatosis (Gougerot-Carteaud): An abnormal host reaction to Malassezia furfur. Acta Derm Venereol (Stockh) 1973; 53: 381 - 384

${ }^{23}$ Humbert P, Treffel P, Chapuis JF, Buchet S, Derancourt C, Agache P. The tetracyclines in dermatology. J Am Acad Dermatol 1991; 25: 691 - 697

${ }^{24}$ Bruynzeel-Koomen CA, de Witt RF. Confluent and reticulated papillomatosis successfully treated with aromatic etretinate. Arch Dermatol 1984; 120: $1236-1237$

${ }^{25}$ Hagedorn M, Schlachter A. Morphologische Untersuchungen der Papillomatosis confluens et reticularis bei Diabetes mellitus. Dermatologica 1983; 167: $127-134$

${ }^{26}$ Jimbow M, Talpash O, Jimbow K. Confluent et reticulated papillomatosis: clinical, light und electron microscopic studies. Int J Dermatol 1992; 31: 480-483

27 Curth HO. Classification of acanthosis nigricans. Int J Dermatol 1976; 15: $592-593$

${ }^{28}$ Schwartz RA. Acanthosis nigricans. J Am Acad Dermatol 1994; 31: $1-22$ 
${ }^{29}$ Rendon MI, Cruz PD, Sontheimer RD, Bergstresser PR. Acanthosis nigricans: A cutaneous marker of tissue resistance to insulin. J Am Acad Dermatol 1989; 21: 461-469

${ }^{30}$ Waisman M. Cutaneous papillomatosis, pseudoacanthosis nigricans, and benign acanthosis nigricans. South Med 1953; 46: 162-169

31 Sönnichsen N, Klug H, Thormann T. Untersuchungen der Morphologie und nosologischen Stellung der Papillomatosis confluens et reticularis (Gougerot-Carteaud). Dermatol Monatschr 1978; 164: 1-15

32 Hamilton D, Tavafoghi V, Schafer JC, Hambrik GW. Confluent and reticulated papillomatosis of Gougerot and Carteaud. Its relation to other papillomatoses. J Am Acad Dermatol 1980; 2: 401 - 410

${ }^{33}$ Hud JA, Cohen JB, Wagner JM. Cruz PD. Prevalence and significance of acanthosis nigricans in an adult obese population. Arch Dermatol 1992; 128: $941-944$

${ }^{34}$ Baalbaki SA, Malak JA, Al-Khars AA. Confluent et reticulated papillomatosis. Treatment with etretinate. Arch Dermatol 1993; 129: $961-963$

${ }^{35}$ Hirokawa M, Matsumoto M, Iizuka H. Confluent et reticulated papillomatosis: A case with concurrent acanthosis nigricans associated with obesity and insulin resistance. Dermatology 1994; 188: 148-151

${ }^{36}$ Hodge JA, Ray MC. Confluent and reticulated papillomatosis: Response to isotretinoin. J Am Acad Dermatol 1991; 24: 654

\section{Preisausschreibung}

\section{Deutscher Hautkrebspreis 2004}

Der Preis in Höhe von 10000 Euro wird in diesem Jahr anlässlich der ISDS/VOD-Tagung im Oktober 2004 in Frankfurt am Main für hervorragende wissenschaftliche Arbeiten in der klinischen und experimentellen Hautkrebsforschung aus dem deutschsprachigen Raum verliehen. Kriterien sind die wissenschaftliche Originalität und Qualität zukunftsweisender Arbeiten in der Dermatoonkologie. Der Preis wird von der Deutschen Hautkrebsstiftung mit finanzieller Unterstützung der fördernden Industrie vergeben. Über die Vergabe entscheidet eine unabhängige Jury von Experten.

Die Bewerbung erfolgt mit Einreichung publizierter Arbeiten (maximal drei) aus den letzten 18 Monaten. Eine schriftliche Zustimmung aller Autoren ist erforderlich. Bei Mehrfachbewerbungen aus einer Klinik ist ein Votum des(r) zuständigen Direktors(in) erwünscht.

Annahmeschluss ist der 30. Juni 2004.

Bewerber (Erstautor, Seniorautor oder Arbeitsgruppe) richten ihre Unterlagen in 10-facher Ausfertigung an:

Deutsche Hautkrebsstiftung

c/o Frau Dr. M. Sommerlad

Zentrum der Dermatologie und Venerologie

Klinikum der J. W. Goethe-Universität

Theodor-Stern-Kai 7

60590 Frankfurt a. M. 